\title{
Viscoelastic Characterization of Short Fibres Reinforced Thermoplastic in Tension and Shearing
}

\author{
A. Andriyana ${ }^{1, a}$, L. Silva ${ }^{1, b}$ and N. Billon ${ }^{2, c}$ \\ ${ }^{1}$ Dep. Of Mech Eng., Univ. Malaya, 56603 Kuala Lumpur, Malaysia \\ ${ }^{2}$ MINES-ParisTech CEMEF, BP 20706904 Sophia Antipolis, France \\ aandri.andriyana@um.edu.my, ${ }^{b}$ Luisa.Silva@mines-paristech.fr, ${ }^{c}$ noelle.billon@mines-paristech.fr
}

Keywords: Short fibres composites; Non-linear behaviour; Video extensometer; Tension; Sheraing.

Abstract. The present work can be regarded as a first step toward an integrated modelling of mould filling during injection moulding process of polymer matrix composites and the resulting material behaviour under service loading conditions. More precisely, the emphasis of the present research is laid on the development of a mechanical model which takes into account the processing-induced microstructure and is capable to predict the mechanical response of the material. In the Part I, a set of experiments which captures the mechanical behaviour of an injection moulded short fibre reinforced under different strain histories is described. Three mechanical testing are conducted: Dynamic Mechanical Analysis (DMA), uniaxial tension and simple shear. Tests show that the material exhibits complex responses mainly due to non-linearity, anisotropy, time/rate-dependence, hysteresis and permanent strain. Moreover, the relaxed state of the material is characterized by the existence of a so-called anisotropic equilibrium hysteresis independently of the prescribed strain rate.

\section{Introduction}

$360 \times 100 \times 3 \mathrm{~mm}^{3}$ plates of a thermoplastic polyamide reinforced by 20 and $30 \mathrm{wt} \%$ of short glass fibres are used in this study. At the macroscopic level, such fibre reinforced thermoplastics are known to exhibit strong directional dependencies, inelastic responses such as time/rate-dependence, temperature dependence, hysteresis and permanent strain (see for example the work of [1-3]).

To gain additional insight into the time-dependent anisotropic behaviour of the composite of interest in this study, experiments probing the mechanical behaviour are conducted using machined samples.

The first step consists in assessing for the mechanical heterogeneities of the plates due to injection moulding to draw some guidelines for samples machining. This is achieved by means of the Dynamic Mechanical Analysis (DMA).

As plates appeared to be homogenous at the macroscopic scale, i.e. no significant variations of the averaged mechanical properties over plate thickness for different location in the injected plates larger scale mechanical testing (uniaxial extension and simple shear) are conducted. For larger strain tension and shearing are performed.

\section{Low strain viscoelasticity and plate homogeneity}

Homogeneity of the injection moulded plate can be illustrated through a set of DMA testing conducted on $45 \times 8 \mathrm{~mm}^{2}$ rectangle-shape specimens of $3 \mathrm{~mm}$ in thickness machined from the plate at different locations as depicted in Fig. 1. DMA is performed in 3 points flexural loading mode for three different frequencies: $0.1,1$ an $10 \mathrm{~Hz}$. Two orientations are retained: perpendicular and parallel to the flow direction during injection process. During the DMA test, the storage modulus, the loss modulus and the loss factor of the specimen are measured as a function of temperature. The temperature is varied from 20 to $110^{\circ} \mathrm{C}$ using a ramp set to $2{ }^{\circ} \mathrm{C} / \mathrm{min}$. 
From these analyses (Fig. 2) it can be seen that loss modulus is not zero indicating that inelastic effects are not neglect able in this material. Moreover, the two modules depend on frequency (i.e. strain rate) over the entire temperature range. In fact, $\alpha$ transition of the composite takes place at 55 ${ }^{\circ} \mathrm{C}$ so that the composite a visco-elastic body close to room temperatureS. This will make strain rate a important parameter to analyse.
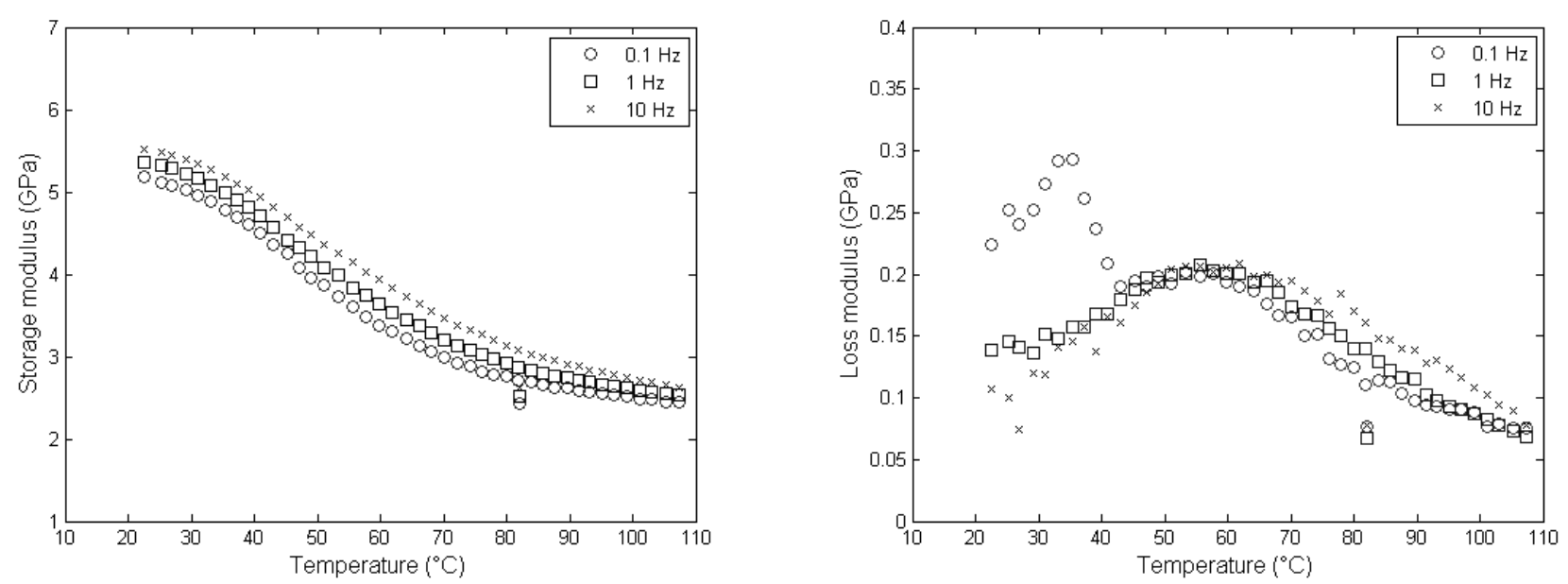

Fig. 2: Evolution of the modulus of a composite with $20 \mathrm{wt} \%$ of fibres in the flow direction as a function of temperature for different frequencies. Left) Storage modulus; Right) Loss modulus.
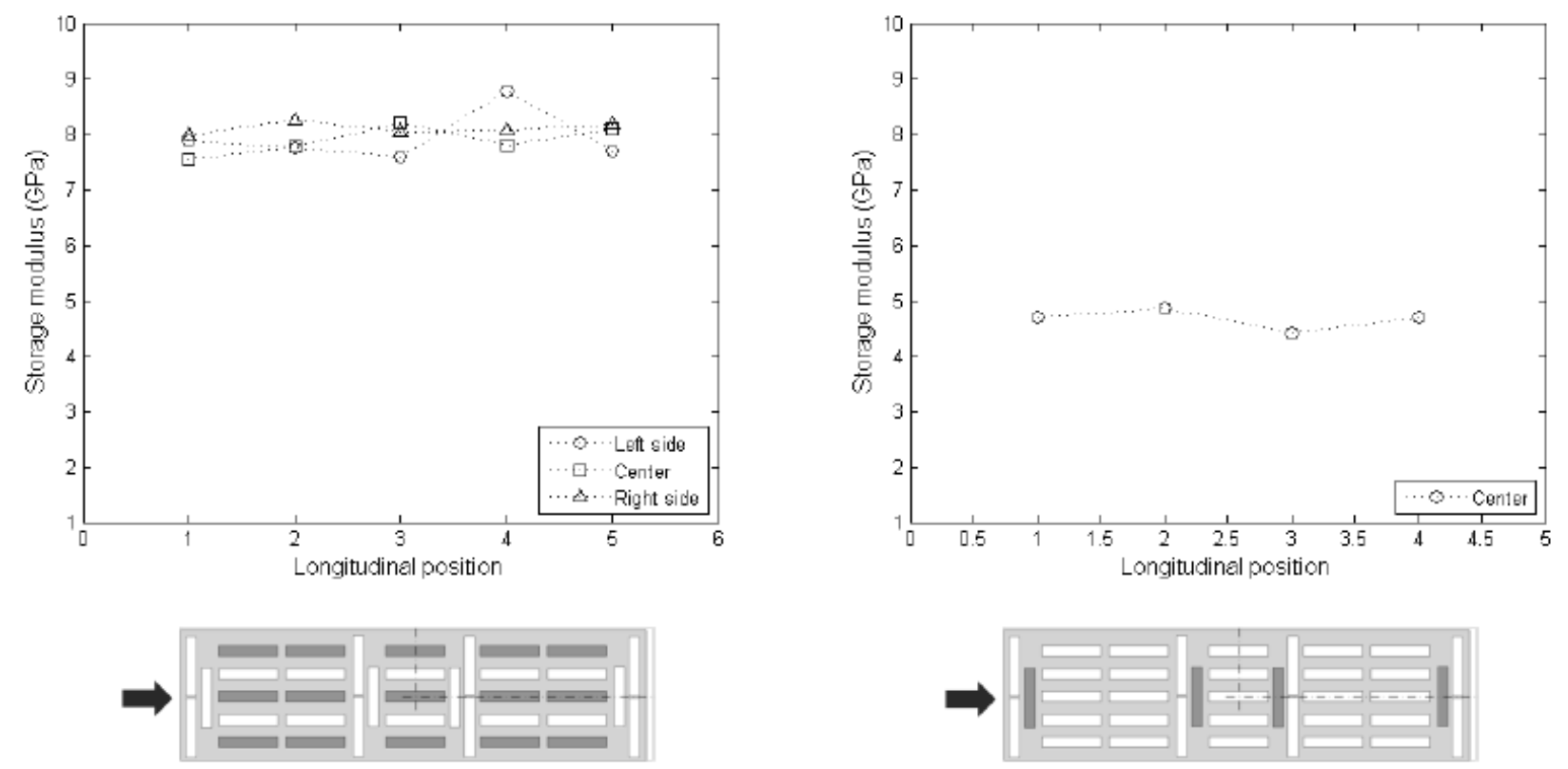

Fig. 1: Evolutions of the storage modulus at temperature of $20^{\circ} \mathrm{C}$ as a function of specimen longitudinal positions in the plate. The arrow denotes the flow direction during injection process. Left) Storage modulus in the flow direction, plotted for three lateral sides called Left, Centre and Right. Right: Storage modulus perpendicular to the flow direction, plotted only for Centre side. The prescribed frequency is $1 \mathrm{~Hz}$. The tested specimens contain $30 \mathrm{wt} \%$ of short fibre and are illustrated by dark coloured specimens on the plates.

Plates can be reasonable assumed to be homogeneous (Fig. 2). Conversely modules parallel and perpendicular to the flow direction are different (see modules on the right-Fig.1 and on the leftFig.1). This will be confirmed with shearing tests. 


\section{Tension and shearing}

Specimens were obtained by machining the plates to get a final geometry as illustrated in Fig. 3 . All tests are carried out at room temperature and the specimens are subjected to various strain histories. Stresses measured in this work are presented in normalized values unless otherwise specified.

In case of tension a video extensometer is used to measure true strain in the central zone. Some 3D measurements allow assessing for compressibility of the material under loading. This is done measuring the 3 strain components.

In case of shearing a full 2D strain field analysis is performed using image correlation and random pattern. This latter is done using paints (Fig. 4). To be complete shear test is performed following Iosipescu (1967) and Walrath and Adams (1983) fixtures [4, 5].

Different orientations of test specimen are used and referred to with their angle with respect to the injection direction (Fig. 5). Using strain field measurements it is possible to check whether strain field are correct or not (i.e., true shearing) in any case. This only ensures that overall strain is close to pure shearing in the ligament. It is than assumed that loading is pure shearing.

However, it is demonstrated that the shear strain fields of specimens having different orientations are not identical suggesting that fibre orientation modifies the strain field in the material under loading. To further investigate the local strain field, the evolutions of the local quantities during monotonic simple shear for specimens having different orientations are plotted: lateral displacement (along v2) and shear strain. To this end, the lateral displacement across the middle longitudinal section of the specimen is plotted for different specimen orientation as depicted in Fig. 6. The displacement is plotted at time $75 \mathrm{~s}$ and $135 \mathrm{~s}$. At time $75 \mathrm{~s}$, the lateral displacement varies linearly independently of the specimen orientation. However, the role of the specimen orientation, i.e. fibre orientation, becomes more significant at higher prescribed displacement. In fact this displacement field gives access to the shear strain field since the latter is obtained from the slope of the former.
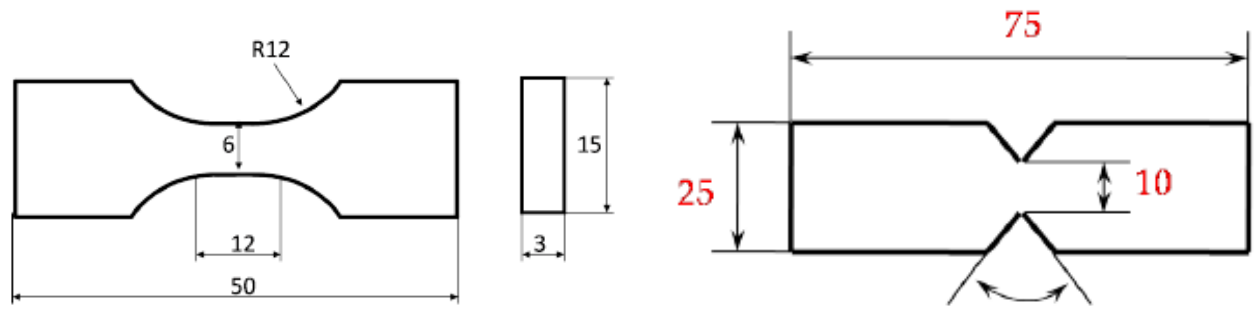

$90^{\circ}$

Fig. 3: Specimen for tension tests (left) and shearing tests (right). All dimensions are in mm.
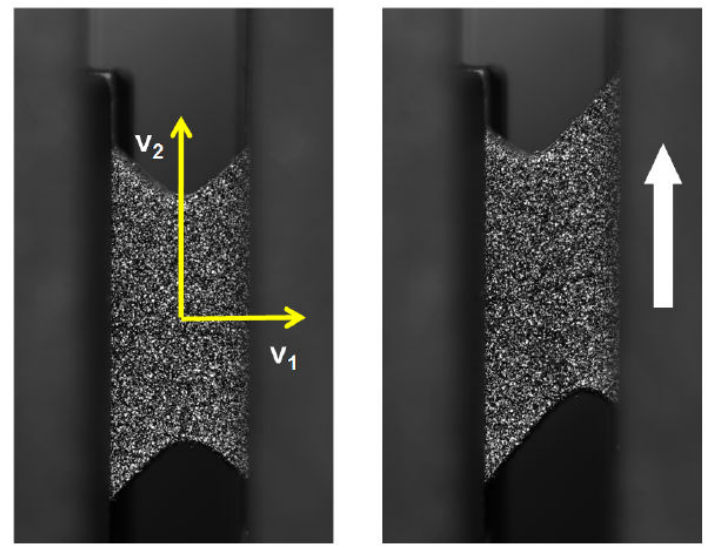

Fig. 4: Undeformed (left) and deformed (right) states of the specimen in shear test. 


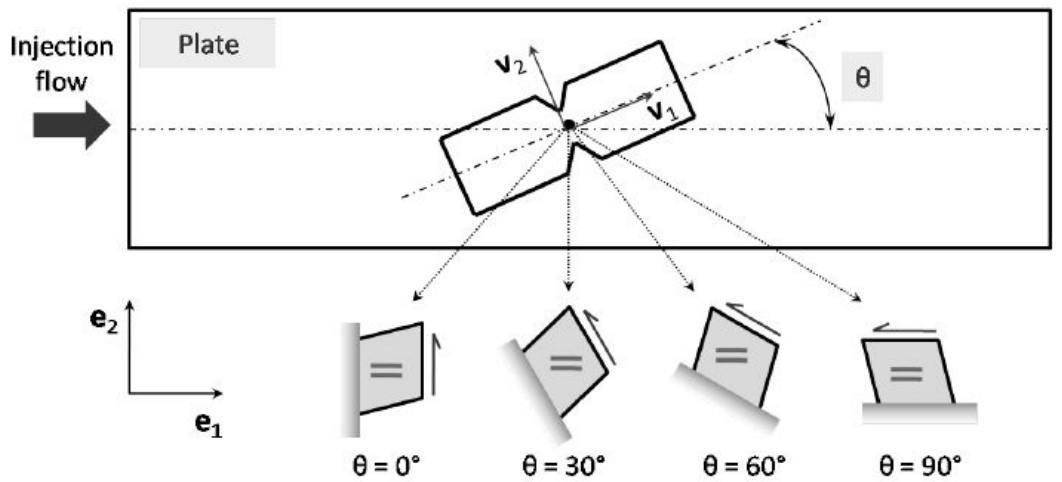

Fig. 5: Different orientations of simple shear test specimen machined from an injection moulded plate.
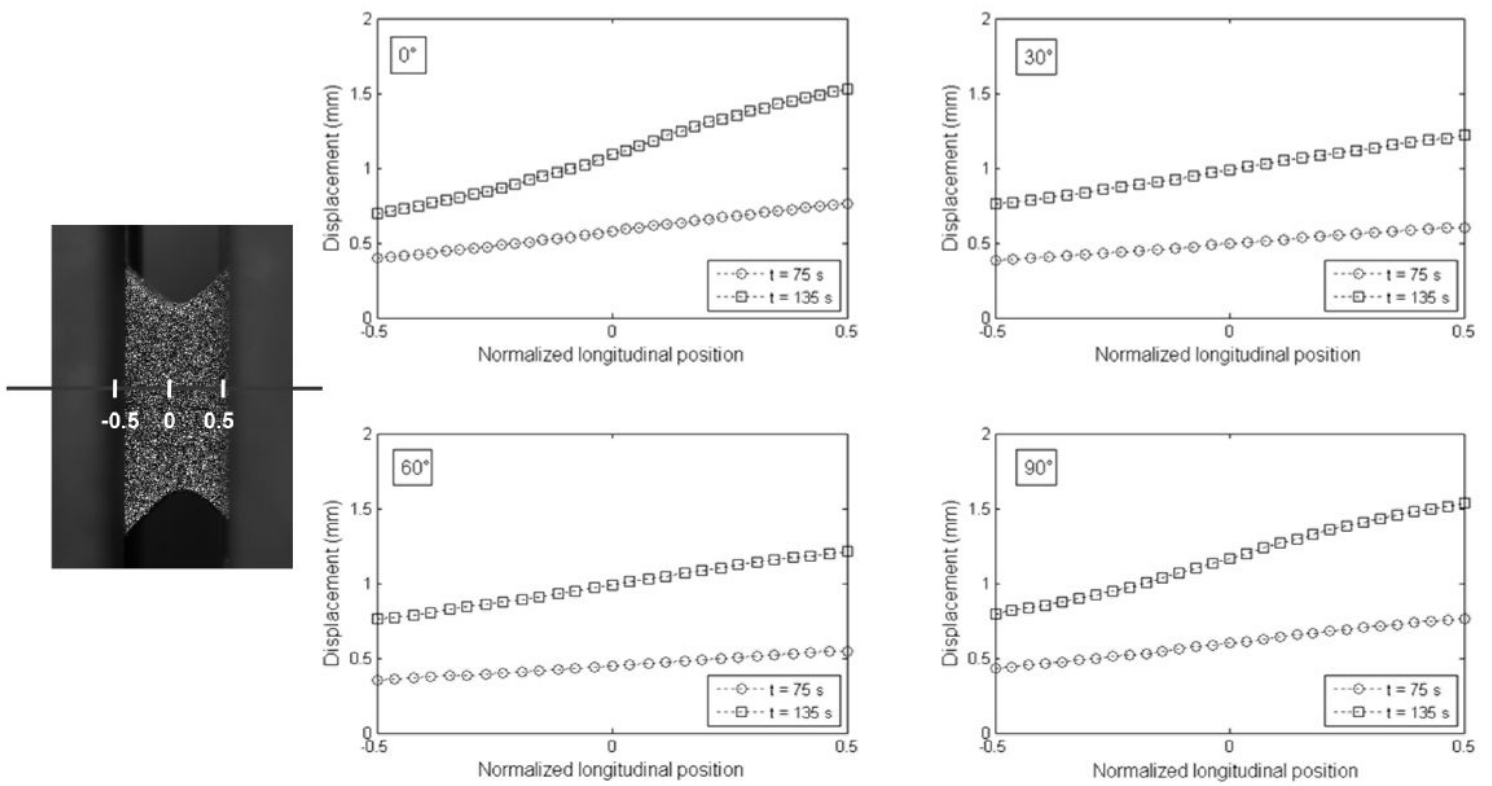

Fig. 6: Evolution of displacement across the middle longitudinal (horizontal) section at time stages of 15 and 27 ( $75 \mathrm{~s}$ and $135 \mathrm{~s}$ respectively) for specimens having different orientations.

Tests consist of loading - unloading tests at different strain rate and up to different totals strain (Fig. 7). This enables to emphasise the visco elastic characteristic of the material.
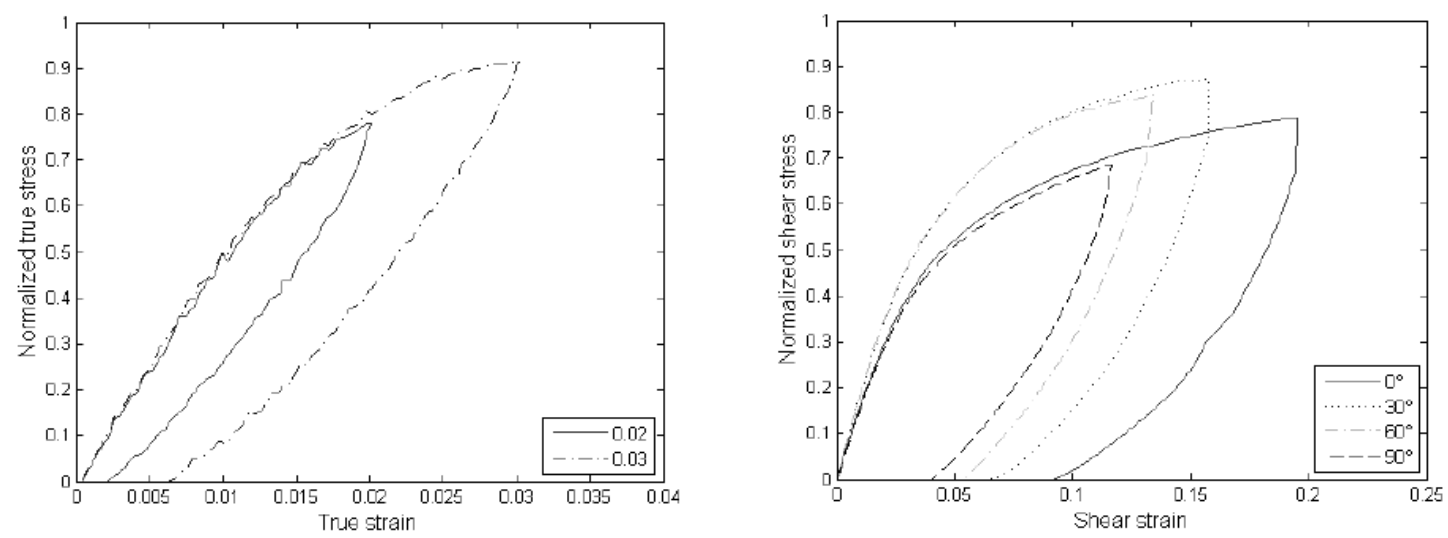

Fig. 7: Upload-unload curves for different maximum strains. Strain rates are $10^{-4} \mathrm{~s}^{-1}$ during upload and $10^{-4} \mathrm{~s}^{-1}$ during unload. Left in tension tests; right in shearing test.

Evidence for a limiting hysteresis loop, i.e. permanent strain, is deduced from tests combining loading and relaxation steps followed be unloading and relaxations step (Fig. 8). 

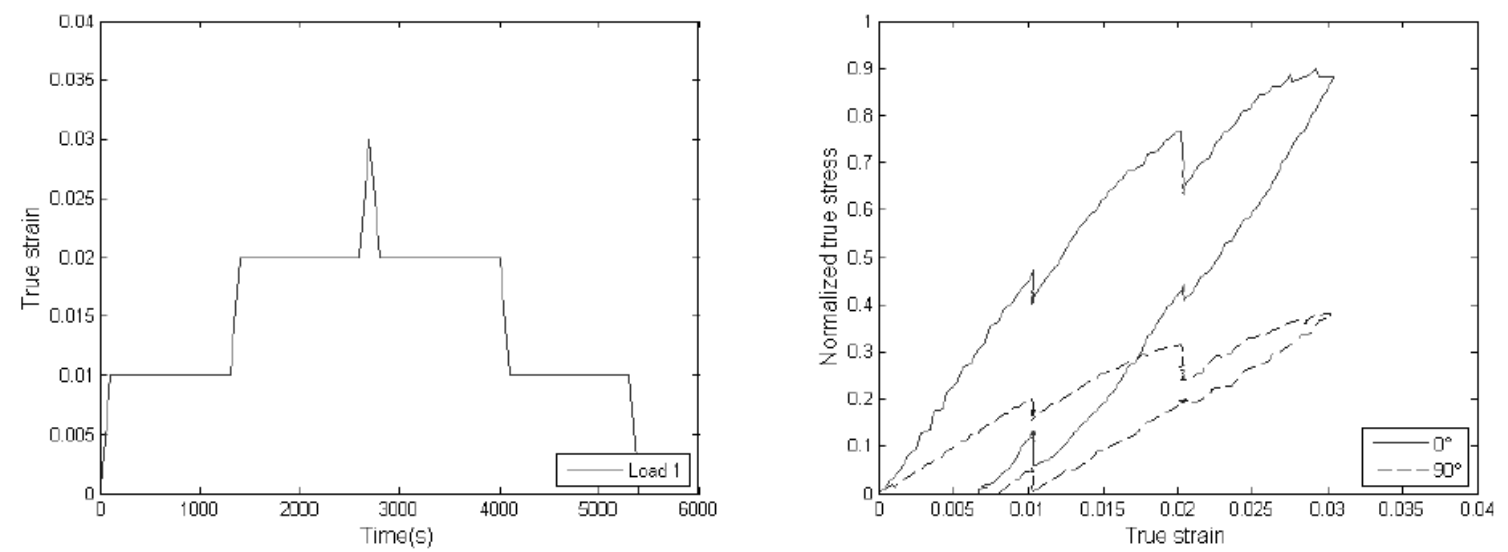

Fig. 8: Uploading and unloading test interrupted by several relaxations illustrating the anisotropic nature of the equilibrium hysteresis. Left: Prescribed strain. Right: Stress response.

\section{Conclusions}

To summarise, the experimental investigation has shown that:

1. No significant heterogeneity in the plate is observed.

2. The material is slightly compressible. This was obviously not decduced from shearing tets but from tensile tests using 3D extensometer (not described here).

3. The material responses are strongly anisotropic and non-linear.

4. The time-dependent behaviour is clearly marked. At relatively small strain, the dependence during the unloading is higher than during the uploading. An opposite trend is observed for higher strain.

5. The existence of a unique equilibrium state cannot be verified using our experimental time scale. Instead, the relaxed state is characterized by an equilibrium hysteresis. Furthermore, the size of the equilibrium hysteresis depends on the fibre content and orientation.

6. Since the equilibrium hysteresis is anisotropic, the total response (stress) cannot be modelled by a simple summation of an isotropic viscoplastic behaviour of the ground matrix and the anisotropic elastic behaviour of the fibres.

\section{References}

[1] Schapery, R. A: Stress analysis of viscoelastic composite materials (Technomic Publishing Co., London, UK 1968).

[2] C. A. Weeks and C. T. Sun: Compos. Sci. Technol. Vol 58 (1998), p. 603

[3] S. V. Thiruppukuzhi and C. T. Su,: Compos. Sci. Technol. Vol. 61 (2001), p. 1

[4] N. Iosipescu J.Mat. Vol. 2(3) (1967), p.537

[5] D. E. Walrath and D. F. Adams Exp. Mech. Vol. 23(1) (1983), p. 105 